\title{
Application of Eckart-Hellmann potential to study selected diatomic molecules using Nikiforov-Uvarov-Functional analysis method
}

\author{
E. P. Inyang ${ }^{a, b, *}$, E. S. William ${ }^{b}$, E. Omugbe ${ }^{c}$, E. P. Inyang ${ }^{b}$, E. A. Ibanga ${ }^{a}$, F. Ayedun ${ }^{a}$, I. O. Akpan ${ }^{b}$, and J. E. Ntibi ${ }^{b}$ \\ ${ }^{a}$ Department of Physics, National Open University of Nigeria, Jabi, Abuja. \\ ${ }^{b}$ Theoretical Physics Group, Department of Physics, University of Calabar, \\ P.M.B 1115, Calabar, Nigeria. \\ ${ }^{c}$ Department of Physics, Federal University of Petroleum Resources, \\ Effurun, Delta State, Nigeria. \\ *e-mail: etidophysics@gmail.com; einyang@noun.edu.ng
}

Received 3 September 2021; accepted 21 September 2021

\begin{abstract}
The energy levels of the Schrödinger equation under the Eckart-Hellmann potential (EHP) energy function are studied by the NikiforovUvarov-Functional Analysis (NUFA) method. We obtained the analytic solution of the energy spectra and the wave function in closed form with the help of Greene-Aldrich approximation. The numerical bound states energy for various screening parameters at different quantum states and vibrational energies of $\mathrm{EHP}$ for $\mathrm{CuLi}, \mathrm{TiH}, \mathrm{VH}$, and TiC diatomic molecules were computed. Four particular cases of this potential were achieved. To test the accuracy of our results, we computed the bound states energy eigenvalues of Hellmann potential which are in excellent agreement with the report of other researchers.
\end{abstract}

Keywords: Schrödinger equation; Nikiforov-Uvarov-Functional Analysis (NUFA) method; Eckart-Hellmann potential; diatomic molecule; Greene-Aldrich approximation.

DOI: https://doi.org/10.31349/RevMexFis.68.020401

\section{Introduction}

The analytical methods for solving bound state problems that arise in physics and their applications have received much attention over the years. The development of these methods allows one to derive the analytic eigen-solutions of the relativistic and non-relativistic wave equations which play a crucial role in interpreting the behavior of quantum mechanical systems. The frequently used analytical methods are the Nikiforov-Uvarov method (NU) [1-30], Asymptotic iterative method (AIM) [31], Laplace transformation approach [32], ansatz solution method [33], super-symmetric quantum mechanics approach (SUSYQM) [34,35], exact and proper quantization methods [36,37], the series expansion method [38-45], and the recent study via the Heun function approach has been used widely to study those soluble quantum systems which could not be solved before, such as the systems including the Mathieu potential, rigid rotor problem, sextictype problem, or the Konwent potential, to name a few [4654].

The Schrödinger equation (SE) can be studied for different quantum-mechanical processes with the above analytical methods [55-58]. The analytical solutions to this equation with a physical potential play an important role in our understanding of the foundations of a quantum system. This is because the eigenvalues and eigenfunctions contain vital information concerning the quantum system under study $[59,60]$. However, the exact bound state solutions of the SE of a number of these potentials are attainable in some cases for example, Coulomb potential [61]. To obtain the approximate solutions when the arbitrary angular momentum quantum number $l$ is not equal to zero, one can solve the SE utilizing a reasonable approximation scheme like the Pekeris or Greene and Aldrich, among others [62-67].

The Eckart potential [68], presented by Eckart in 1930, is a diatomic molecular potential model. Because of its significance in physics and chemical physics, numerous authors in have considered the bound state solutions of the wave equations for this potential; see [69-74] and references therein.

The Hellmann potential [75] has been widely utilized by numerous authors to obtain bound state solutions in atomic, nuclear and particle physics [76-79], and applications in condensed matter physics [80].

Recent studies have focused on the combination of, at least, two potentials. The essence of combining two or more physical potential models is to take into account more physical phenomena into existing investigations of molecular physics [81-85].

With this in mind, we explore the approximate bound state analytical solutions to the SE with the Eckart plus Hellmann potential using Nikiforov-Uvarov-Functional Analysis (NUFA) method. The obtained energy equation will be applied to study the energy spectra of some selected diatomic molecules. The combined potential takes the form $[68,75]$

$$
V(r)=-\frac{A e^{-\alpha r}}{1-e^{-\alpha r}}+\frac{B e^{-\alpha r}}{\left(1-e^{-\alpha r}\right)^{2}}-\frac{C}{r}+\frac{D e^{-\alpha r}}{r},
$$

where $A, B, C$, and $D$ are the strength of the potential, $\alpha$ is the screening parameter and $r$ is inter molecular distance.

The paper is organized as follows: In Sec. 2, a brief introduction of the NUFA method is presented. In Sec. 3 we solve the SE with the EHP to obtain the energy equation and wave function. In Sec. 4, the derived energy equation will be used 
to obtain the numerical computation of energy eigenvalues at different states and selected diatomic molecules. In Sec. 4, we present the results and discussion. Conclusions are given in Sec. 5.

\section{Nikiforov-Uvarov-functional (NUFA) method}

analysis

Using the concepts of the NU, parametric NU and the functional analysis methods [1,86,87], Ikot et al. [88] proposed a simple and elegant method for solving a second order differential equation of the hypergeometric type called NikiforovUvarov-Functional Analysis method (NUFA) method. This method is easy and simple. The NU method is used to solve a second-order differential equation of the form [1]

$$
\psi^{\prime \prime}(z)+\frac{\tilde{\tau}(z)}{\sigma} \psi^{\prime}(z)+\frac{\tilde{\sigma}(z)}{\sigma^{2}(z)} \psi(z)=0,
$$

where $\tilde{\sigma}(z)$ are $\sigma(z)$ polynomials of, at most, second degree and $\tilde{\tau}(z)$ is a polynomial of, at most, fist degree. Tezcan and Sever [86] latter introduced the parametric form of NU method in the form

$$
\begin{aligned}
\psi^{\prime \prime} & +\frac{\alpha_{1}-\alpha_{2} z}{z\left(1-\alpha_{3} z\right)} \psi^{\prime}+\frac{1}{z^{2}\left(1-\alpha_{3} z\right)^{2}} \\
& \times\left(-\xi_{1} z^{2}+\xi_{2} z-\xi_{3}\right) \psi(s)=0,
\end{aligned}
$$

where $\alpha_{1}$ are $\xi_{i}(i=1,2,3)$ are all parameters. It can be observed in Eq. (3) that the differential equation has two singularities at $z \rightarrow 0$ and $z \rightarrow 1$, thus we take the wave function in the form,

$$
\psi(z)=z^{\lambda}(1-z)^{\nu} f(z)
$$

Substituting Eq. (4) into Eq. (3) leads to the following equation,

$$
\begin{aligned}
z(1-\alpha z) f^{\prime \prime}(z)+ & \left(\alpha_{1}+2 \lambda-\left[2 \lambda \alpha_{3}+2 \nu \alpha_{3}+\alpha_{2}\right] z\right) f^{\prime}(z)-\alpha_{3}\left(\lambda+\nu+\frac{\alpha_{2}}{\alpha_{3}}-1+\sqrt{\left[\frac{\alpha_{2}}{\alpha_{3}}-1\right]^{2}+\frac{\xi_{1}}{\alpha_{3}}}\right) \\
\times & \left(\lambda+\nu+\frac{\alpha_{2}}{\alpha_{3}^{2}}-1+\sqrt{\left.\left[\frac{\alpha_{2}}{\alpha_{3}}-1\right]^{2}+\frac{\xi_{1}}{\alpha_{3}^{2}}\right)+\left(\alpha_{2} \nu-\alpha_{1} \alpha_{3} \nu+\nu(\nu-1) \alpha_{3}\right.}\right. \\
& \left.\times \frac{\lambda(\lambda-1)+\alpha_{1} \lambda-\xi_{3}}{z}+\frac{-\frac{\xi_{1}}{\alpha_{3}}+\xi_{2}-\xi_{3} \alpha_{3}}{\left(1-\alpha_{3} z\right)}\right) f(z)=0 .
\end{aligned}
$$

Equation (5) can be reduced to a Gauss hypergeometric equation if and only if the following conditions are satisfied:

$$
\begin{array}{r}
\lambda(\lambda-1)+\alpha_{1} \lambda-\xi_{3}=0, \\
\alpha_{2} \nu-\alpha_{1} \alpha_{3} \nu+\nu(\nu-1) \alpha_{3}-\frac{\xi_{1}}{\alpha_{3}}+\xi_{2}-\xi_{3}=0 .
\end{array}
$$

Thus, Eq. (5) becomes

$$
\begin{aligned}
z\left(1-\alpha_{1} z\right) f^{\prime \prime}(z) & +\left(\alpha_{1}+2 \lambda-\left[2 \lambda \alpha_{3}+2 \nu \alpha_{3}+\alpha_{2}\right] z\right) f^{\prime}(z)-\alpha_{3}\left(\lambda+\nu+\frac{\alpha_{2}}{\alpha_{3}}-1+\sqrt{\left[\frac{\alpha_{2}}{\alpha_{3}}-1\right]^{2}+\frac{\xi_{1}}{\alpha_{3}}}\right) \\
& \times\left(\lambda+\nu+\frac{\alpha_{2}}{\alpha_{3}^{2}}-1+\sqrt{\left[\frac{\alpha_{2}}{\alpha_{3}}-1\right]^{2}+\frac{\xi_{1}}{\alpha_{3}^{2}}}\right) f(z)=0 .
\end{aligned}
$$

Solving Eqs. (6) and (7) gives Eqs. (9) and (10),

$$
\begin{aligned}
\lambda & =\frac{\left(1-\alpha_{1}\right)}{2} \pm \frac{1}{2} \sqrt{\left(1-\alpha_{1}\right)^{2}+4 \xi_{3}}, \\
\nu & =\frac{\left(\alpha_{3}+\alpha_{1} \alpha_{3}-\alpha_{2}\right) \pm \sqrt{\left(\alpha_{3}+\alpha_{1} \alpha_{3}-\alpha_{2}\right)^{2}+\left(\frac{\xi_{1}}{\alpha_{3}}+\alpha_{3} \xi_{3}-\xi_{2}\right)}}{2} .
\end{aligned}
$$

Equation (8) is the hyper geometric equation of the form,

$$
x(1-x) f^{\prime \prime}(x)+(c+[a+b+1] x) f^{1}(x)-a b f(x)=0 .
$$


Using Eqs. (4),(8) and (11), we obtain the energy equation and the corresponding wave equation, respectively, for the NUFA method as follows:

$$
\begin{aligned}
\lambda^{2}+ & 2 \lambda\left(\nu+\frac{\alpha_{2}}{\alpha_{1}}-1+\frac{n}{\sqrt{\alpha_{3}}}\right)+\left(\nu+\frac{\alpha_{2}}{\alpha_{3}}-1+\frac{n}{\sqrt{\alpha_{3}}}\right)^{2}-\left(\frac{\alpha_{2}}{\alpha_{3}}-1\right)^{2}-\frac{\xi_{1}}{\alpha_{3}^{2}}=0, \\
\psi(z)= & N_{z} \frac{\left(1-\alpha_{1}\right)+\sqrt{\left(1-\alpha_{1}\right)^{2}+4 \xi_{3}}}{2}\left(1-\alpha_{3} z\right) \\
& \times \frac{\left(\alpha_{3}+\alpha_{1} \alpha_{3}-\alpha_{2}\right) \pm \sqrt{\left(\alpha_{3}+\alpha_{1} \alpha_{3}-\alpha_{2}\right)^{2}+\left(\frac{\xi_{1}}{\alpha_{3}^{2}+\alpha_{3} \xi_{3}-\xi_{2}}\right)}}{2}{ }_{2} F_{1}(a, b, c, z),
\end{aligned}
$$

where $a, b$, are $c$ given as follows;

$$
\begin{aligned}
& a=\sqrt{\alpha_{3}}\left(\lambda+\nu+\frac{\alpha_{2}}{\alpha_{3}}-1+\sqrt{\left[\frac{\alpha_{2}}{\alpha_{3}}-1\right]^{2}+\frac{\xi_{1}}{\alpha_{3}}}\right), \\
& b=\sqrt{\alpha_{3}}\left(\lambda+\nu+\frac{\alpha_{2}}{\alpha_{3}}-1-\sqrt{\left[\frac{\alpha_{2}}{\alpha_{3}}-1\right]^{2}+\frac{\xi_{1}}{\alpha_{3}}}\right), \\
& c=\alpha_{1}+2 \lambda .
\end{aligned}
$$

\section{Approximate solutions of the Schrödinger equation with Eckart plus Hellmann potential}

The SE takes the form [2]

$$
\frac{d^{2} U(r)}{d r^{2}}+\left(\frac{2 \mu}{\hbar^{2}}\left[E_{n l}-V(r)\right]-\frac{l(l+1)}{r^{2}}\right) U(r)=0,
$$

where $E_{n l}$ is the energy eigenvalues of the quantum system, $l$ is the angular momentum quantum number, $\mu$ is the reduced mass of the system, $\hbar$ is the reduced Planck's constant and $r$ is radial distance from the origin.

Equation (17) cannot be solved exactly with the proposed potential. So we introduce an approximation scheme proposed by Greene-Aldrich [62] to deal with the centrifugal barrier. This approximation is a good approximation to the centrifugal term which is valid for $\alpha \ll 1$, and it becomes

$$
\frac{1}{r^{2}} \approx \frac{\alpha^{2}}{\left(1-e^{-\alpha r}\right)}
$$

Substituting Eqs. (1) and (18) into Eq. (17) and (19) yields

$$
\frac{d^{2} U(r)}{d r^{2}}+\left(\frac{2 \mu}{\hbar^{2}}\left[E_{n l}-\frac{A e^{-\alpha r}}{1-e^{-\alpha r}}-\frac{B e^{-\alpha r}}{\left\{1-e^{-\alpha r}\right\}^{2}}+\frac{C \alpha}{1-e^{-\alpha r}}-\frac{D e^{-\alpha r}}{1-e^{-\alpha r}}\right]+\frac{l(l+1) \alpha^{2}}{\left[1 e^{-\alpha r}\right]^{2}}\right) U(r)=0,
$$

which can be further simplified using the change of variables given by

$$
x=e^{-\alpha r},
$$

for which we get

$$
\frac{d^{2} U(x)}{d x^{2}}+\frac{1-x}{x(1-x)} \frac{d U(x)}{d x}+\frac{1}{x^{2}(1-x)^{2}}\left(-\left[\varepsilon-\beta_{0}-\beta_{3}\right] x^{2}+\left[2 \varepsilon-\beta_{0}+\beta_{1}-\beta_{2}-\beta_{3}\right] x-\left[\varepsilon-\beta_{2}+\gamma\right]\right) U(x)=0,
$$

where

$$
-\varepsilon=\frac{2 \mu E_{n l}}{\alpha^{2} \hbar^{2}}, \quad \beta_{0}=\frac{2 \mu A}{\alpha^{2} \hbar^{2}}, \quad \beta_{1}=\frac{2 \mu B}{\alpha^{2} \hbar^{2}}, \quad \beta_{2}=\frac{2 \mu C}{\alpha^{2} \hbar^{2}}, \quad \beta_{3}=\frac{2 \mu D}{\alpha^{2} \hbar^{2}}, \quad \gamma=l(l+1) .
$$

Upon comparing Eqs. (3) and (21), we obtain the relevant polynomials as:

$$
\alpha_{1}=\alpha_{2}=\alpha_{3}=1, \quad \xi_{1}=\varepsilon-\beta_{0}-\beta_{3}, \quad \xi_{2}=2 \varepsilon-\beta_{0}+\beta_{1}-\beta_{2}-\beta_{3}, \quad \xi_{3}=\varepsilon-\beta_{2}+\gamma .
$$


Inserting the polynomials given by Eq. (23) into Eqs. (9) and (10), we have

$$
\begin{array}{r}
\lambda=\sqrt{4\left(\varepsilon-\beta_{2}+\gamma\right)}, \\
\nu=\frac{1}{2} \pm \sqrt{1+4\left(\gamma-\beta_{1}\right)} .
\end{array}
$$

Substituting Eqs. (22-25) into Eqs. (12), we obtain the energy equation

$$
\begin{aligned}
E_{n l} & =\frac{\alpha^{2} \hbar^{2}\left(l+l^{2}\right)}{2 \mu}-A-C \alpha-\frac{\hbar^{2} \alpha^{2}}{8 \mu}\left(\left[n+\frac{1}{2}+\sqrt{\frac{1}{4}+\frac{2 B \mu}{\alpha^{2} \hbar^{2}}+\left\{l+l^{2}\right\}}\right]^{2}\right. \\
& \left.\times \frac{-\frac{2 A \mu}{\alpha^{2} \hbar^{2}}+\frac{2 B \mu}{\alpha^{2} \hbar^{2}}-\frac{2 C \mu}{\alpha \hbar^{2}}+\frac{2 D \mu}{\alpha \hbar^{2}}+\left[l+l^{2}\right]}{n+\frac{1}{2}+\sqrt{\frac{1}{4}+\frac{2 B \mu}{\alpha^{2} \hbar^{2}}+\left[l+l^{2}\right]}}\right)^{2} \cdot
\end{aligned}
$$

which is in agreement with Eq. (38) of Ref. [77].

\subsection{Particular case}

To test for the accuracy of our results, we set some parameters in Eqs. (1) and (26) to zero and obtain four particular cases of potential and energy equation.

1. We set $A=B=0$ and obtain the Hellmann potential and its energy equation, respectively, as

$$
\begin{aligned}
& V(r)=-\frac{C}{r}+\frac{D e^{-\alpha r}}{r}, \\
& E_{n l}=\frac{\alpha^{2} \hbar^{2}\left(l+l^{2}\right)}{2 \mu}-C \alpha-\frac{\alpha^{2} \hbar^{2}}{8 \mu}\left(\frac{\left[n+\frac{1}{2}+\sqrt{\frac{1}{4}+\left(l+l^{2}\right)}\right]^{2}-\frac{2 C \mu}{\alpha \hbar^{2}}+\frac{2 D \mu}{\alpha \hbar^{2}}+\left(l+l^{2}\right)}{n+\frac{1}{2}+\sqrt{\frac{1}{4}+\left(l+l^{2}\right)}}\right)^{2} .
\end{aligned}
$$

2. We set $C=D=0$ and obtain the Eckart potential and its energy equation, respectively, as

$$
\begin{aligned}
V(r) & =-\frac{A e^{-\alpha r}}{1-e^{-\alpha r}}+\frac{B e^{-\alpha r}}{\left(1-e^{-\alpha r}\right)^{2}} . \\
E_{n l} & =\frac{\alpha^{2} \hbar^{2}\left(l+l^{2}\right)}{2 \mu}-A-\frac{\hbar^{2} \alpha^{2}}{8 \mu}\left(\frac{\left[n+\frac{1}{2}+\sqrt{\frac{1}{4}+\frac{2 B \mu}{\alpha^{2} \hbar^{2}}+\left(l+l^{2}\right)}\right]^{2}-\frac{2 A \mu}{\alpha^{2} \hbar^{2}}+\frac{2 B \mu}{\alpha^{2} \hbar^{2}}+\left(l+l^{2}\right)}{n+\frac{1}{2}+\sqrt{\frac{1}{4}+\frac{2 B \mu}{\alpha^{2} \hbar^{2}}+\left(l+l^{2}\right)}}\right)^{2} .
\end{aligned}
$$

3. We set $A=B=D=\alpha=0$ and obtain the Coulomb potential and its energy equation, respectively, as

$$
\begin{aligned}
V(r) & =-\frac{C}{r}, \\
E_{n l} & =-\frac{\mu C^{2}}{2 \hbar^{2}\left(n_{-} r+l+1\right)^{2}},
\end{aligned}
$$

where $n_{-} r+l+1=n$ is the principal quantum number.

The result of Eq. (32) is consistent with the result obtained in Eq. (36) in Ref. [21].

$$
\begin{aligned}
& V(r)=-\frac{D e^{-\alpha r}}{r} \\
& E_{n l}=-\frac{\alpha^{2} \hbar^{2}\left(l+l^{2}\right)}{2 \mu}-\frac{\hbar^{2} \alpha^{2}}{8 \mu}\left(\frac{\left[n+\frac{1}{2}+\sqrt{\frac{1}{4}+\left(l+l^{2}\right)}\right]^{2}+\frac{2 D \mu}{\alpha \hbar^{2}}+\left(l+l^{2}\right)}{n+\frac{1}{2}+\sqrt{\frac{1}{4}+\left(l+l^{2}\right)}}\right)^{2} .
\end{aligned}
$$


TABLE I. Bound state energy eigenvalues $(\mathrm{eV})$ of the Eckart-Hellmann potential with $\hbar=\mu=1$.

\begin{tabular}{|c|c|c|c|c|}
\hline State & $\alpha$ & $\begin{array}{c}A=0.01, B=0.5 \\
C=1, D=-1\end{array}$ & $\begin{array}{c}A=0.005, B=0.25 \\
C=2, D=-2\end{array}$ & $\begin{array}{c}A=0.0025, B=0.125, \\
C=4, D=-4\end{array}$ \\
\hline \multirow[t]{5}{*}{$1 \mathrm{~s}$} & 0.025 & -0.5263521625 & -0.3104395873 & -0.3041019021 \\
\hline & 0.050 & -0.5563594522 & -0.3923969578 & -0.6301704138 \\
\hline & 0.075 & -0.5898519860 & -0.4938113093 & -1.081923010 \\
\hline & 0.100 & -0.6266198628 & -0.6127403461 & -1.640204160 \\
\hline & 0.150 & -0.709198628 & -0.8960512898 & -3.009546904 \\
\hline \multirow[t]{5}{*}{$2 \mathrm{~s}$} & 0.025 & -0.5278462720 & -0.3134850809 & -0.3064758005 \\
\hline & 0.050 & -0.5622260539 & -0.4015176484 & -0.6147211738 \\
\hline & 0.075 & -0.6021935108 & -0.5081767351 & -1.004551117 \\
\hline & 0.100 & -0.6469257426 & -0.6289588255 & -1.445599734 \\
\hline & 0.150 & -0.7480659625 & -0.8999476994 & -2.405459656 \\
\hline \multirow[t]{5}{*}{$2 p$} & 0.025 & -0.5257258961 & -0.3097912239 & -0.3031052053 \\
\hline & 0.050 & -0.5538354335 & -0.3895266085 & -0.6221624598 \\
\hline & 0.075 & -0.5841024422 & -0.4863981654 & -1.050909737 \\
\hline & 0.100 & -0.6162307409 & -0.5974170774 & -1.557847309 \\
\hline & 0.150 & -0.6848372934 & -0.8500952692 & -2.695002862 \\
\hline \multirow[t]{5}{*}{$3 \mathrm{~s}$} & 0.025 & -0.5298392842 & -0.3168335532 & -0.3091243145 \\
\hline & 0.050 & -0.5696640174 & -0.4113242511 & -0.6050340761 \\
\hline & 0.075 & -0.6173762645 & -0.5240248324 & -0.9557836844 \\
\hline & 0.100 & -0.6713937028 & -0.6488631591 & -1.331376322 \\
\hline & 0.150 & -0.7939098630 & -0.9203977116 & -2.099855330 \\
\hline \multirow[t]{5}{*}{$3 p$} & 0.025 & -0.5272195493 & -0.3128354399 & -0.3055088368 \\
\hline & 0.050 & -0.5596966036 & -0.3986642162 & -0.6077677944 \\
\hline & 0.075 & -0.5964276548 & -0.5009934106 & -0.9806451254 \\
\hline & 0.100 & -0.6365166896 & -0.6146925374 & -1.388641432 \\
\hline & 0.150 & -0.7238605000 & -0.8612543564 & -2.224098580 \\
\hline \multirow[t]{5}{*}{$3 d$} & 0.025 & -0.5244733721 & -0.3084948329 & -0.3011225088 \\
\hline & 0.050 & -0.5487880544 & -0.3838064758 & -0.606747212 \\
\hline & 0.075 & -0.5726108402 & -0.4717847684 & -0.9945628778 \\
\hline & 0.100 & -0.5954927171 & -0.5678320312 & -1.419102816 \\
\hline & 0.150 & -0.6365278213 & -0.7672835076 & -2.251887755 \\
\hline \multirow[t]{5}{*}{$4 \mathrm{~s}$} & 0.025 & -0.5323000579 & -0.3204668349 & -0.3120277371 \\
\hline & 0.050 & -0.5785157811 & -0.4218033616 & -0.5996463159 \\
\hline & 0.075 & -0.6350697965 & -0.5413329210 & -0.9254852509 \\
\hline & 0.100 & -0.6995504645 & -0.6720273560 & -1.263244881 \\
\hline & 0.150 & -0.8462213682 & -0.9524357106 & -1.935504414 \\
\hline \multirow[t]{5}{*}{$4 p$} & 0.025 & -0.5292122102 & -0.3161831200 & -0.3081839761 \\
\hline & 0.050 & -0.5671318525 & -0.4084922092 & -0.5988699390 \\
\hline & 0.075 & -0.6116097382 & -0.5170512012 & -0.9364831796 \\
\hline & 0.100 & -0.6610134522 & -0.6354309818 & -1.288956165 \\
\hline & 0.150 & -0.7700398596 & -0.8863990934 & -1.980709322 \\
\hline
\end{tabular}



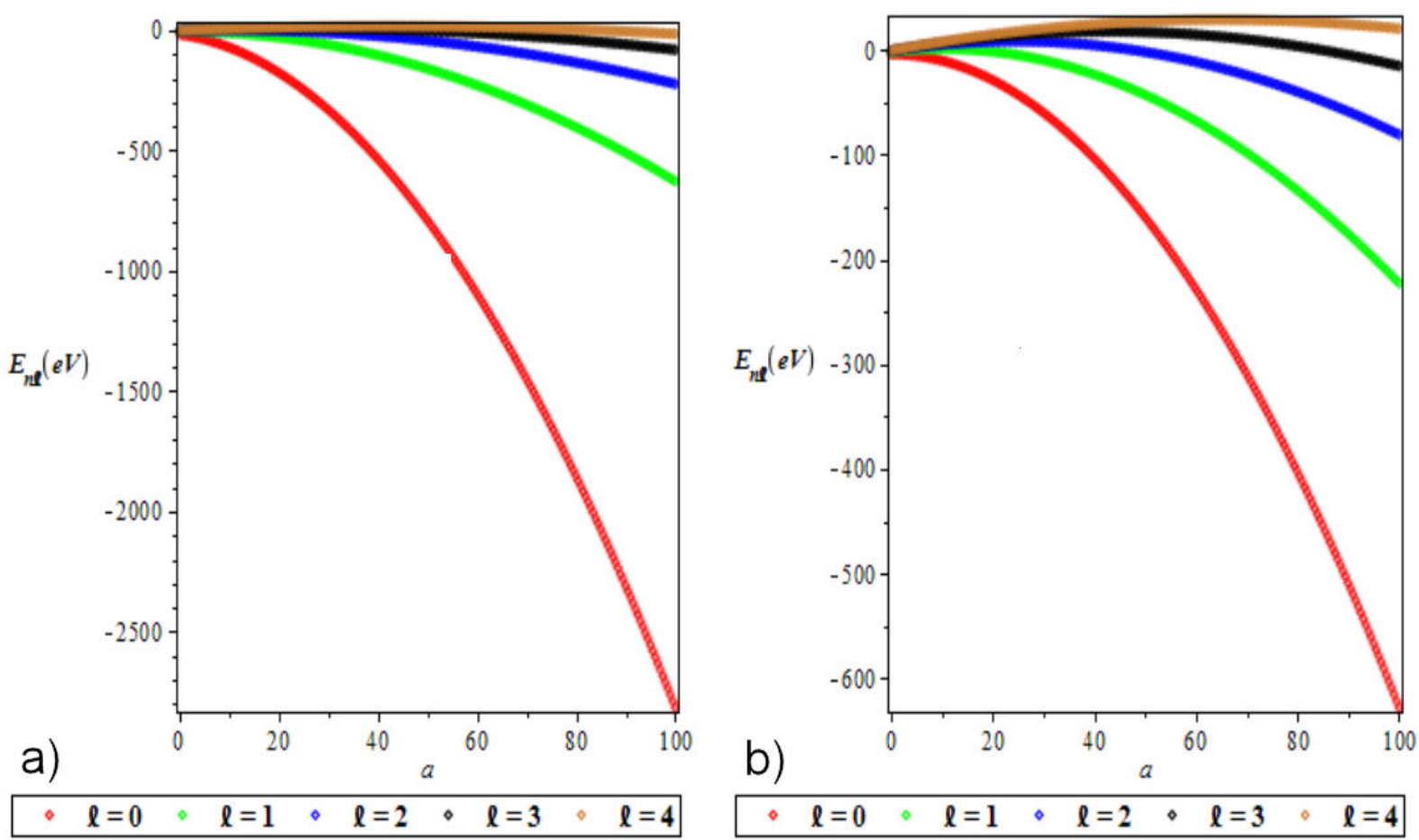

FIGURE 1. a) Variation of the ground state energy spectra for different values of $l$ as a function of $a$ and b) The plot of the first excited state energy spectra for different $l$ as a function of $a$. We choose $A=1, B=-1, C=4, D=-4$ and $\alpha=0.025$ for the ground and excited states.
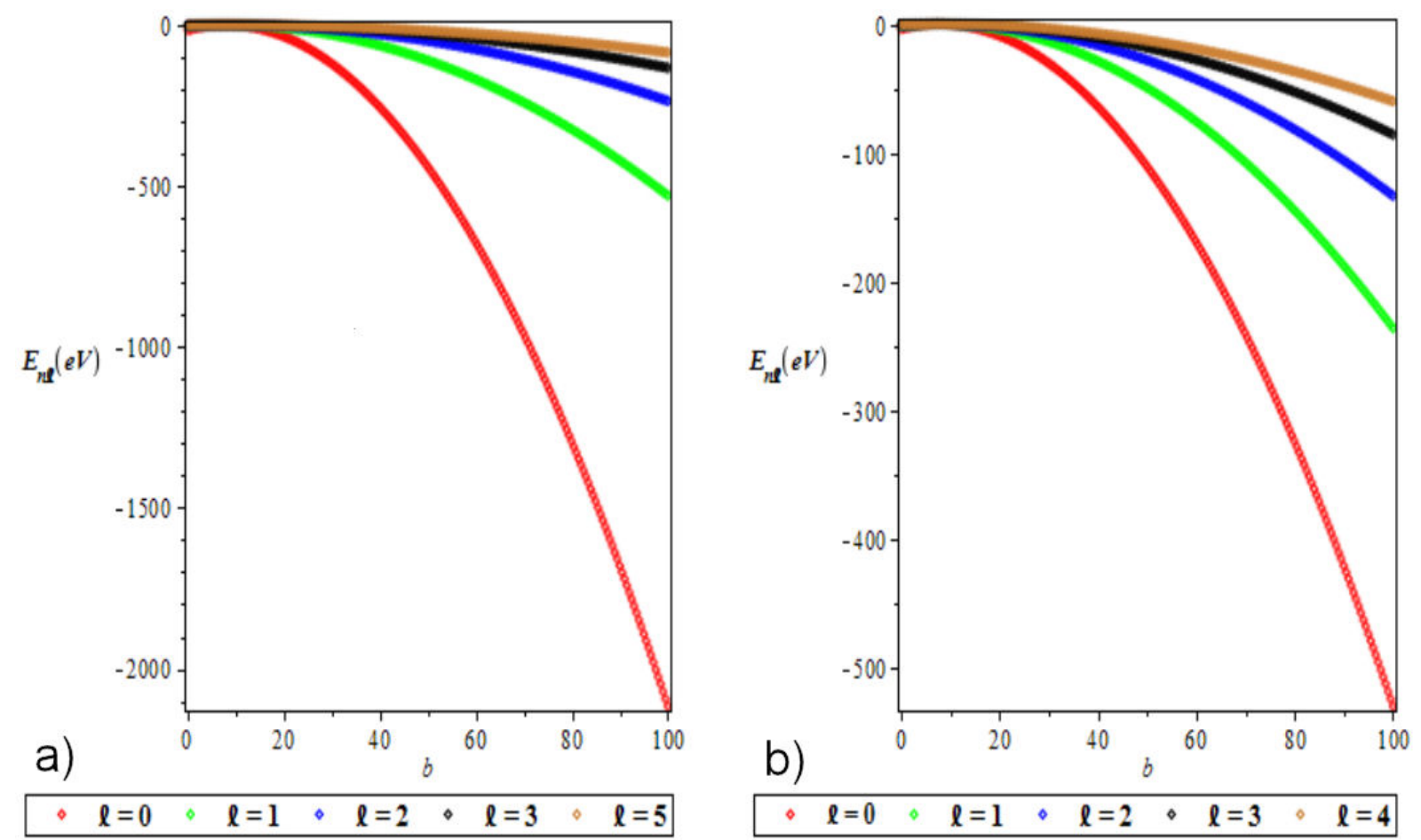

FIGURE 2. a) Variation of the ground state energy spectra for different values of $l$ as a function of $b$ and b) The plot of the first excited state energy spectra for various $l$ as a function of $b$. We choose $A=1, B=-1, C=4, D=-4$ and $\alpha=0.025$ for the ground and excited states. 

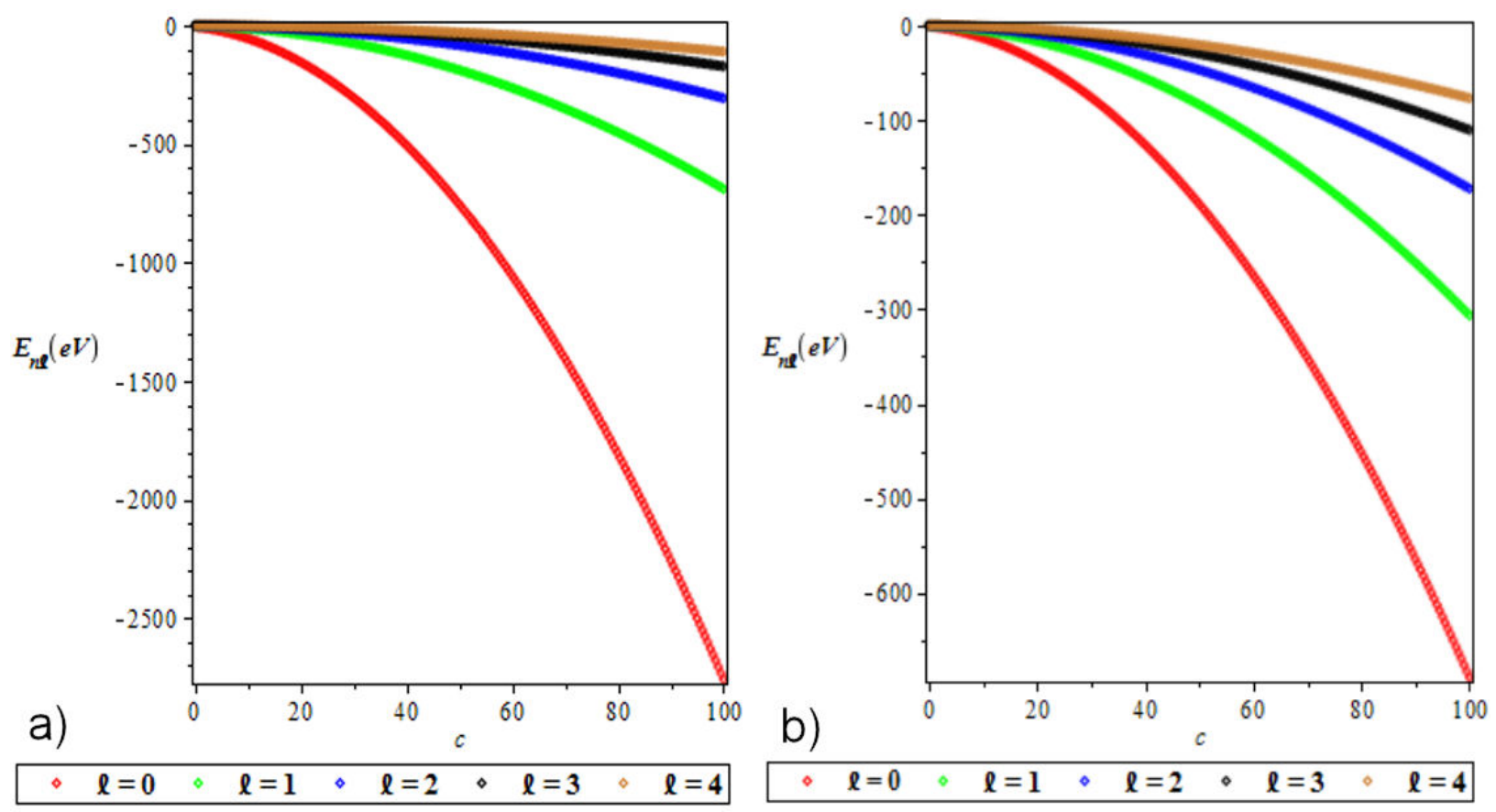

FIgURE 3. a) Variation of the ground state energy spectra for different values of $l$ as a function of $c$ and b) The plot of the first excited state energy spectra for various $l$ as a function of $c$. We choose $A=1, B=-1, C=4, D=-4$ and $\alpha=0.025$ for the ground and excited states.
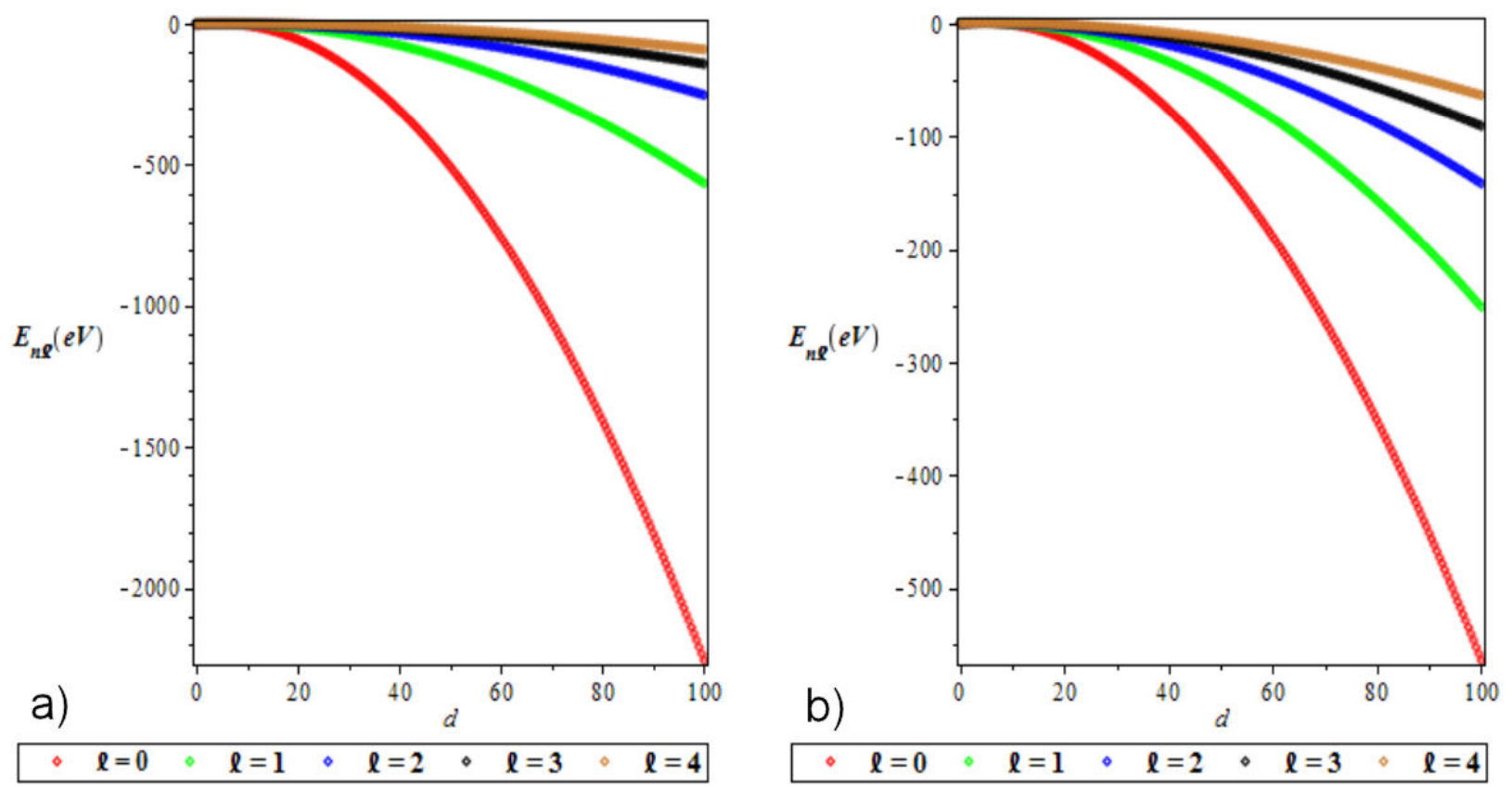

FIgURE 4. a) Variation of the ground state energy spectra for different values of $l$ as a function of $d$ and b) The plot of the first excited state energy spectra for different values of $l$ as a function of $d$. We choose $A=1, B=-1, C=4, D=-4$ and $\alpha=0.025$ for the ground and excited states. 

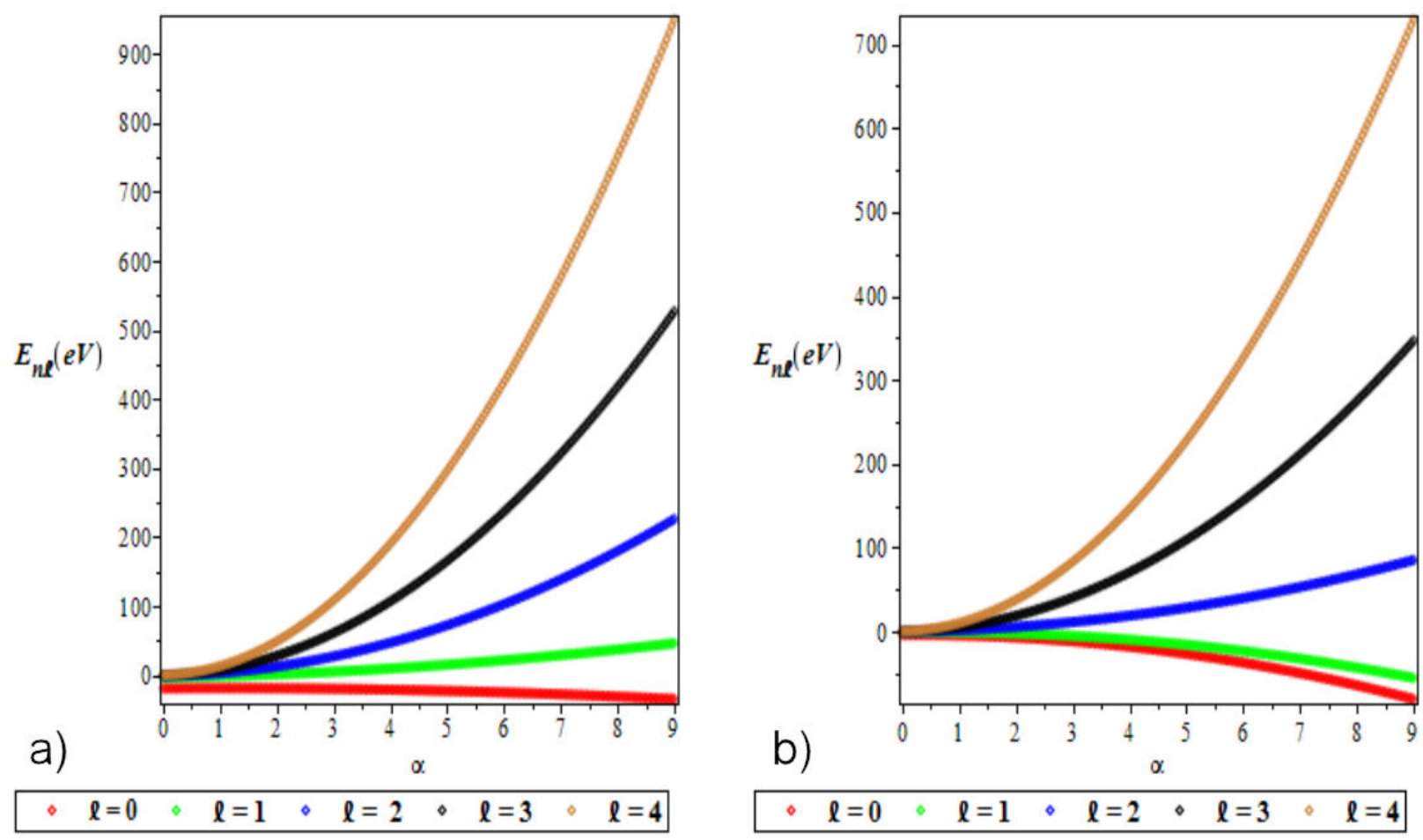

FIGURE 5. a) Variation of the ground state energy spectra for different values of $l$ as a function of the screening parameter $\alpha$ and b) Plot of the first excited state energy spectra for different $l$ as a function of the screening parameter $\alpha$. We choose $A=1, B=-1, C=4, D=-4$ and $\alpha=0.025$ for the ground and excited states

The result of Eq. (34) is consistent with the result obtained in Eq. (38) in Ref. [24].

The corresponding wavefunction is given as

$$
\psi(x)=N \frac{\sqrt{4\left(\varepsilon-\beta_{2}+\gamma\right)}}{2}(1-z) \sqrt{4\left(4\left[\gamma-\beta_{1}\right]\right)_{2}} F_{1}(a, b, c ; z),
$$

where

$$
\begin{aligned}
& a=\sqrt{4\left(\varepsilon-\beta_{2}+\gamma\right)}+\frac{1}{2} \pm \sqrt{1+4\left(\gamma-\beta_{1}\right)}+\sqrt{\varepsilon-\beta_{0}-\beta_{3}}, \\
& b=\sqrt{4\left(\varepsilon-\beta_{2}+\gamma\right)}+\frac{1}{2} \pm \sqrt{1+4\left(\gamma-\beta_{1}\right)}-\sqrt{\varepsilon-\beta_{0}-\beta_{3}}, \\
& c=1+2 \sqrt{4\left(\varepsilon-\beta_{2}+\gamma\right)} .
\end{aligned}
$$

\section{Results and discussion}

To test the accuracy of our results, we computed the bound states energy eigenvalues of EHP with $\hbar=\mu=1$ using arbitrary potential parameters as presented in Table I. The results show that as the screening parameter and potential strength increase there is a decrease in energy eigenvalues at different quantum states. We apply the experimental data obtained from Ref. [89] as presented in Table II and used the conversions: $1 \mathrm{amu}=931.494028 \mathrm{MeV} / \mathrm{c}^{2}$ and $\hbar c=1973.29 \mathrm{eV} \AA$ [91] to compute the vibrational energies of EHP for $\mathrm{CuLi}$, $\mathrm{TiH}, \mathrm{VH}$, and $\mathrm{TiC}$ diatomic molecules using Eq. (26). The numerical computation is given in Table III. It is observed that for each vibrational quantum number, the vibrational energies increase with increase in the rotational quantum number, for each of the selected diatomic molecules. The num- merical energy eigenvalues for Hellmann potential are also computed to check for the accuracy of the NUFA method as presented in Table IV. The results are in good agreement with the earlier studies of Ref. [76] with the NU, AP, and SUSY methods of Ref. [77] and the PT method of [78].

TABLE II. Parameters of selected diatomic molecules used in this study $[89,90]$.

\begin{tabular}{cccc}
\hline Molecules & $\mu(\mathrm{MeV})$ & $\alpha\left(\AA^{-1}\right)$ & $\mu(\mathrm{amu})$ \\
\hline $\mathrm{VH}$ & 0.09203207571 & 1.44370 & 0.988005 \\
$\mathrm{TiH}$ & 0.09197301899 & 1.32408 & 0.987371 \\
$\mathrm{TiC}$ & 0.8948005221 & 1.52550 & 9.606079 \\
$\mathrm{CuLi}$ & 0.58306812793 & 1.00818 & 6.259494 \\
\hline
\end{tabular}


TABLE III. Bound state energy spectra $E_{n l}(\mathrm{eV})$ of the Eckart plus Hellmann potential for VH, TiH, TiC and CuLi diatomic molecules

\begin{tabular}{|c|c|c|c|c|c|}
\hline$n$ & $l$ & $E_{n l}(\mathrm{eV})$ of $\mathrm{VH}$ & $E_{n l}(\mathrm{eV})$ of $\mathrm{TiH}$ & $E_{n l}(\mathrm{eV})$ of $\mathrm{TiC}$ & $E_{n l}(\mathrm{eV})$ of $\mathrm{CuLi}$ \\
\hline 0 & 0 & -4.388393324 & -3.995461324 & -4.687262254 & -3.014649864 \\
\hline 0 & 1 & -4.363576420 & -3.976563694 & -4.684013156 & -3.013313002 \\
\hline 0 & 2 & -4.315482766 & -3.939708307 & -4.677541581 & -3.010644546 \\
\hline 0 & 3 & -4.246835875 & -3.886588443 & -4.667899981 & -3.006654929 \\
\hline 0 & 4 & -4.160974946 & -3.819343691 & -4.655165101 & -3.001359541 \\
\hline 0 & 5 & -4.061278595 & -3.740228732 & -4.639435781 & -2.994778429 \\
\hline 1 & 0 & -4.351517256 & -3.974290474 & -4.665595573 & -3.015347165 \\
\hline 1 & 1 & -4.329538507 & -3.957243671 & -4.662501668 & -3.014042854 \\
\hline 1 & 2 & -4.286754140 & -3.923883123 & -4.656337945 & -3.011439114 \\
\hline 1 & 3 & -4.225253356 & -3.875536963 & -4.647151876 & -3.007545617 \\
\hline 1 & 4 & -4.147627936 & -3.813899190 & -4.635012960 & -3.002376632 \\
\hline 1 & 5 & -4.056543954 & -3.740778524 & -4.620010768 & -2.995950756 \\
\hline 2 & 0 & -4.330562184 & -3964109406 & -4.646784709 & -3.016648024 \\
\hline 2 & 1 & -4.310755837 & -3.948515786 & -4.643830791 & -3.015373953 \\
\hline 2 & 2 & -4.272055607 & -3.917910429 & -4.637944814 & -3.012830346 \\
\hline 2 & 3 & -4.216096257 & -3.873352684 & -4.629169881 & -3.009026184 \\
\hline 2 & 4 & -4.144926129 & -3.816204629 & -4.617569118 & -3.003974719 \\
\hline 2 & 5 & -4.060681968 & -3.747937031 & -4.603223944 & -2.997693235 \\
\hline 3 & 0 & -4.32179419 & -3.962583306 & -4.630535913 & -3.018511506 \\
\hline 3 & 1 & -4.303698065 & -3.948159012 & -4.627708751 & -3.017265610 \\
\hline 3 & 2 & -4.268227540 & -3.919778876 & -4.622074329 & -3.014778033 \\
\hline 3 & 3 & -4.216682122 & -3.878299314 & -4.613671886 & -3.011057130 \\
\hline 3 & 4 & -4.150704094 & -3.824829161 & -4.602558928 & -3.006115232 \\
\hline 3 & 5 & -4.072027217 & -3.760577424 & -4.588809670 & -2.999968427 \\
\hline 4 & 0 & -4.322668435 & -3.968078354 & -4.616595736 & -3.020901395 \\
\hline 4 & 1 & -4.305952612 & -3.954615747 & -4.613883777 & -3.019681816 \\
\hline 4 & 2 & -4.273099190 & -3.928071892 & -4.608478018 & -3.017246588 \\
\hline 4 & 3 & -4.225154073 & -3.889146536 & -4.600414287 & -3.013603491 \\
\hline 4 & 4 & -4.163448775 & -3.838751163 & -4.589745102 & -3.008764019 \\
\hline 4 & 5 & -4.089403533 & -3.777888034 & -4.576538290 & -3.002743168 \\
\hline 5 & 0 & -4.331402395 & -3.979423541 & -4.604744582 & -3.023785550 \\
\hline 5 & 1 & -4.315825387 & -3.966767477 & -4.602137686 & -3.022590621 \\
\hline 5 & 2 & -4.285139270 & -3.9051768464 & -4.596940511 & -3.020204428 \\
\hline 5 & 3 & -4.240193658 & -3.905002862 & -4.589185844 & -3.016634230 \\
\hline 5 & 4 & -4.182077111 & -3.857225419 & -4.578921771 & -3.011890752 \\
\hline 5 & 5 & -4.111961137 & -3.799272206 & -4.566210430 & -3.005987997 \\
\hline
\end{tabular}


TABLE IV. Comparison of energy eigenvalues (eV) for a special case of Hellmann potential as a function of the screening parameter $\alpha$ with $\hbar=2 \mu=1$ for $A=B=0, B=2$, and $D=-1$.

\begin{tabular}{|c|c|c|c|c|c|}
\hline State & $\alpha$ & Present method & $(\mathrm{NU})$ [76] & (AP) [77] & (PT) [78] \\
\hline \multirow[t]{3}{*}{$1 \mathrm{~S}$} & 0.001 & -2.250500250 & -2.250500 & -2.248981 & -2.24900 \\
\hline & 0.005 & -2.252506250 & -2.252506 & - 2.244993 & -2.24501 \\
\hline & 0.01 & -2.255025000 & -2.255025 & -2.240030 & -2.24005 \\
\hline \multirow[t]{3}{*}{$2 \mathrm{~S}$} & 0.001 & -0.5630010000 & -0.563001 & -0.561502 & -0.561502 \\
\hline & 0.005 & -0.5650250000 & -0.565025 & -0.557549 & -0.557550 \\
\hline & 0.01 & -0.5676000000 & -0.567600 & -0.552697 & -0.552697 \\
\hline \multirow[t]{3}{*}{$2 \mathrm{P}$} & 0.001 & -0.5622502500 & -0.563000 & -0.561502 & -0.561502 \\
\hline & 0.005 & -0.5612562500 & -0.565000 & - 0.557541 & -0.557541 \\
\hline & 0.01 & -0.5600250000 & -0.567500 & -0.552664 & -0.552664 \\
\hline \multirow[t]{3}{*}{$3 S$} & 0.001 & -0.2505022500 & -0.250502 & -0.249004 & -0.249004 \\
\hline & 0.005 & -0.2525562500 & -0.252556 & -0245110 & -0.245111 \\
\hline & 0.01 & -0.2552250000 & -0.255225 & -0.240435 & -0.240435 \\
\hline \multirow[t]{3}{*}{$3 p$} & 0.001 & -0.2501680278 & -0.250501 & -0.249004 & -0.249004 \\
\hline & 0.005 & -0.2508673611 & -0.252531 & -0.245102 & -0.245103 \\
\hline & 0.01 & -0.2518027778 & -0.255125 & -0.240404 & $-0: 240404$ \\
\hline \multirow[t]{3}{*}{$3 d$} & 0.001 & -0.2495002500 & -0.250833 & -0.249003 & -0.249003 \\
\hline & 0.005 & -0.2475062500 & -0.254151 & -0.245086 & -0.245086 \\
\hline & 0.01 & -0.2450250000 & -0.258269 & -0.240341 & -0.240341 \\
\hline \multirow[t]{3}{*}{$4 \mathrm{~S}$} & 0.001 & -0.1411290000 & -0.141129 & -0.139633 & -0.139633 \\
\hline & 0.005 & -0.1432250000 & -0.143225 & -0.135819 & -0.135819 \\
\hline & 0.01 & -0.1460250000 & -0.146025 & -0.131380 & -0.131381 \\
\hline \multirow[t]{3}{*}{$4 p$} & 0.001 & -0.1409405625 & -0.141128 & -0.139632 & 0.139633 \\
\hline & 0.005 & -0.1422640625 & -0.143200 & -0.135811 & 0.135811 \\
\hline & 0.01 & -0.1440562500 & -0.145925 & -0.131350 & -0.131351 \\
\hline \multirow[t]{3}{*}{$4 d$} & 0.001 & -0.1405640625 & -0.141314 & -0.139632 & -0.139632 \\
\hline & 0.005 & -0.1403515625 & -0.144089 & -0.135795 & -0.135796 \\
\hline & 0.01 & -0.1401562500 & -0.147606 & -0.131290 & -0.131290 \\
\hline \multirow[t]{3}{*}{$4 f$} & 0.001 & -0.1400002500 & -0.141686 & -0.139631 & -0.139631 \\
\hline & 0.005 & -0.1375062500 & -0.145902 & -0.135772 & -0.135772 \\
\hline & 0.01 & -0.1344000000 & -0.151106 & -0.131200 & -0.131200 \\
\hline
\end{tabular}

In Figs. 1-4, we plotted the ground and excited states energy eigenvalues of the different quantum states as a function of the EHP strengths, respectively. We observed that there is a decrease in energy in both the ground and excited states as the potential strength, parametrized by $A, B, C$, and $D$, increases In Fig. 5a) and 5b), we plotted the energy eigenvalues of EHP versus the screening parameter. Here, the energy increases for $\ell=0,1,2$ and 3 in the ground states and decreases in $\ell=4$, as the screening parameter increases. We also observed increase in energy for $\ell=0,1$ and 2 decrease in $\ell=3$ and 4 as the screening parameter increases in the excited states.

\section{Conclusion}

In this article, the bound state solutions to the Schrödinger equation with EHP have been studied within the GreeneAldrich approximation scheme. The eigenvalues and the eigen functions are obtained using the NUFA method. We then apply the energy equation for four diatomic molecules by using the experimental values of each molecular parameter. The results show that the bound state energy spectra of these diatomic molecules increases as various quantum numbers also increase. To test the accuracy of our results, we computed the bound states energy $(\mathrm{eV})$ eigenvalues of EHP and Hellmann potential, in agreement with previous studies. 
We plotted the ground and excited states energy eigenvalues of the different quantum states as a function of the EHP strengths, respectively. We observed that there is a decrease in energy in both the ground and excited states as the potential strength increases.
1. S.K. Nikiforov, and V.B. Uvarov. Special functions of mathematical Physics (Birkhäuser, Boston, 1988). https://doi. org/10.1007/978-1-4757-1595-8

2. E.P. Inyang, E.P. Inyang, I.O. Akpan, J.E. Ntibi, and E.S. William, Masses and thermodynamic properties of a Quarkonium system, Can. J. Phys. 99 (2021) 982, https : / / doi. org/10.1139/cjp-2020-0578

3. I.O. Akpan, E. P. Inyang, E. P.Inyang, and E. S. William, Approximate solutions of the Schrödinger equation with Hulthén-Hellmann Potentials for a Quarkonium system. Rev. Mex. Fis. 67 (2021) 482, https://doi.org/10.31349/ RevMexFis.67.482

4. E. P. Inyang, E. P. Inyang, J. E. Ntibi, E.E. Ibekwe and E. S. William, Analytical study on the Applicability of Ultra Generalized Exponential Hyperbolic potential to predict the mass spectra of the Heavy Mesons, arXiv:2101.06389.

5. J. E. Ntibi, E. P. Inyang, E. P. Inyang and E. S. William, Relativistic Treatment of D-Dimensional Klien-Gordon equation with Yukawa potential. J. Innov. Sci. Eng. Technol. 7 (2020) 28.

6. M. Abu-Shady, C.O. Edet and A.N. Ikot, Non-relativistic Quark model under external magnetic and Aharanov-Bohm(AB) fields in the Presence of temperature-dependent confined Cornell potential. Can. J. Phys. 99 (2021) 1024, https: //doi .org/ $10.1139 / \mathrm{cjp}-2020-0101$.

7. J. A. Obu, E. S. William, I. O. Akpan, E. A. Thompson, and E. P. Inyang, Analytical Investigation of the Single-Particle Energy Spectrum in Magic Nuclei of ${ }^{56} \mathrm{Ni}$ and ${ }^{116} \mathrm{Sn}$, Eur. J. Appl. Phys. 2 (2020) 1, https://doi.org/10.24018/ ejphysics.2020.2.6.28

8. E. S. William, E. P. Inyang, and E. A. Thompson, Arbitrary solutions of the Schrödinger equation interacting with HulthénHellmann potential model. Rev. Mex. Fisi. 66 (2020) 730, https://doi.org/10.31349/RevMexFis.66.730.

9. C. M. Ekpo et al., New Generalized Morse-Like Potential for studying the atomic interaction in diatomic molecules. arXiv:2012.02581.

10. I. B. Okon et al., Thermodynamic properties and Bound state solutions of Schrödinger equation with Mobius square plus screened-Kratzer potential using Nikiforov-Uvarov method, Comput. Theor. Chem. 1196 (2021) 113132, https://doi. org/10.1016/j.comptc.2020.113132

11. M. Abu-Shady, $N$-dimensional Schrödinger equation at finite temperature using the Nikiforov-Uvarov method. J. Egypt. Math. Soc. 25 (2017) 86, https://doi.org/10.1016/ j.joems.2016.06.006

12. P.O. Okoi, C. O. Edet, and T. O. Magu, Relativistic treatment of the Hellmann generalized Morse potential, Rev. Mex. Fis. 66 (2020) 1, https://doi.org/10.31349/RevMexFis. 66.1
13. C. O. Edet, and P.O. Okoi, Any l-state solutions of the Schrödinger equation for $q$-deformed Hulthén plus generalized inverse quadratic Yukawa potential in arbitrary dimensions, Rev. Mex. Fis. 65 (2019) 333, https://doi.org/ 10.31349/RevMexFis.65.333

14. E. P. Inyang, E. S. William and J.A. Obu, Eigensolutions of the N-dimensional Schrödinger equation interacting with Varshni-Hulthén potential model, Rev. Mexi. Fis. 67 (2021) 193, https://doi.org/10.31349/RevMexFis.67. 193.

15. E. P. Inyang et al., Thermodynamic properties and mass spectra of a quarkonium system with Ultra Generalized ExponentialHyperbolic potential. Commun. Phys. Sci. 7 (2021) 97.

16. E.P. Inyang, B.I. Ita and E.P. Inyang, Relativistic treatment of Quantum mechanical Gravitational-Harmonic Oscillator potential. Eur. J. Appl. Phys. 3 (2021) 42, https://doi.org/ 10.24018/ejphysics.2021.3.3.83

17. E.P. Inyang, E. P. Inyang, J.E. Ntibi, E.E. Ibekwe, and E.S. William, Analytical study on the Applicability of Ultra Generalized exponential Hyperbolic potential to predict the mass spectra of the heavy mesons, arXiv:2101.06389.

18. E.P. Inyang, E.P. Inyang, E. S. William and E.E. Ibekwe, Study on the applicability of Varshni potential to predict the massspectra of the Quark-Antiquark systems in a non-relativistic framework. Jord. J. Phys. 14 (2021) 339.

19. P. Nwabuzor et al., Analyzing the effects of Topological defect(TD) on the Energy Spectra and Thermal Properties of $\mathrm{LiH}, \mathrm{TiC}$ and $\mathrm{I}_{2}$ Diatomic molecules. Entropy 23 (2021) 1060. https://doi.org/10.3390/e23081060

20. E.P. Inyang, E.P. Inyang, J. E. Ntibi, E. E. Ibekwe, and E. S. William, Approximate solutions of D-dimensional KleinGordon equation with Yukawa potential via Nikiforov-Uvarov method, Ind. J. Phys. 95 (2021) 2733, https : / doi .org/ 10.1007/s12648-020-01933-x

21. C. O. Edet, U. S. Okorie, A. T. Ngiangia, and A. N. Ikot, Bound state solutions of the Schrödinger equation for the modified Kratzer plus screened Coulomb potential. Ind. J. Phys. 94 (2020) 425, https://doi.org/10.1007/ s12648-019-01477-9

22. M. Abu-Shady, N-dimensional Schrödinger equation at finite temperature using the Nikiforov-Uvarov method. Journal of Egyptian Mathematical Society 23 (2016) 4.

23. P.O. Okoi, C.O. Edet, and T.O. Magu, Relativistic treatment of the Hellmann generalized Morse potential. Rev. Mex. Fis. 66 (2020) 10 .

24. C. O. Edet, P. O. Okoi, A. S. Yusuf, P. O. Oshie, and P. O. Amadi,. Bound state solutions of the generalized shifted Hulthen potential. Ind. J. Phys 95 (2021) 471, https:// doi.org/10.1007/s12648-019-01650-0. 
25. U. S. Okorie, C. O. Edet, A. N. Ikot, G. J. Rampho, and R. Sever, Thermodynamic function for diatomic molecules with modified Kratzer plus screened Coulomb potential. Ind. J. Phys. 95 (2021) 411, https://doi.org/10.1007/ s12648-019-01670-w

26. E. Omugbe, Non-relativistic eigensolutions of molecular and heavy quarkonia interacting potentials via the NikiforovUvarov method, Can. J. Phys. 98 (2020) 1125, https:// doi.org/10.1139/cjp-2020-0039

27. C.O. Edet, P.O. Okoi, and S.O. Chima, Analytic solutions of the Schrödinger equation with non-central generalized inverse quadratic Yukawa potential, Rev. Bras. Ensino Fis. 42 (2020) e20190083, https : / doi.org/10.1590/ 1806-9126-RBEF-2019-0083

28. L. Hitler ,B.I. Ita, T.O.Magu, O.U.Akakuru, N.A.NzeataIbe, A.I.Ikeuba, A.I.Pigweh, C.O.Edet, Solutions to the Dirac Equation for Manning-Rosen Plus Shifted Deng-Fan Potential and CoulombLike Tensor Interaction Using Nikiforov-Uvarov Method. Intl. J. Chem. 10 (2018) 99, https://doi.org/ 10.5539/ijc.v10n3p99

29. A.I. Ahmadov, C. Aydin, and O. Uzun, Bound state solution of the Schrödinger equation at finite temperature, J.Phys.: Conf. Series 1194 (2019) 012001, https: / / doi.org/10. 1088/1742-6596/1194/1/012001

30. H. Hassanabadi, E. Maghsoodi, A.N. Ikot, and S. Zarrinkamar, Approximate arbitrary-state solutions of Dirac equation for modified deformed Hylleraas and modified Eckart potentials by the NU method. Appl. Math. Comput. 219 (2013) 9388, https://doi.org/10.1016/j.amc.2013.03.011

31. R. Rani, S. B. Bhardwaj, and F. Chand, Mass Spectra of Heavy and Light Mesons Using Asymptotic Iteration Method, Commu. Theo. Phys. 70 (2018) 179, https://doi.org/ $10.1088 / 0253-6102 / 70 / 2 / 179$

32. M. Abu-Shady, T. A. Abdel-Karim, and E. M. Khokha, Exact Solution of the N-dimensional Radial Schrödinger Equation via Laplace Transformation Method with the Generalized Cornell Potential, arXiv:1802.02092.

33. S.H. Dong, The Ansatz Method for Analyzing Schrödinger's Equation with Three Anharmonic Potentials in D Dimensions. J. Genet. Couns. 15 (2002) 385. https://doi.org/10. 1023/A:1021220712636

34. F. Cooper, A. Khare, and U. Sukhatme, Supersymmetry and quantum mechanics, Phys. Rep. 251 (1995) 267, https:// doi.org/10.1016/0370-1573(94)00080-M.

35. M. Abu-Shady and A. N. Ikot, Analytic solution of multidimensional Schrödinger equation in hot and dense QCD media using the SUSYQM method. Eur. Phys. J. Plus 134 (2019) 321, https://doi.org/10.1140/epjp/ i2019-12685-y

36. S.H. Dong, Wave Equations in Higher Dimensions (Springer, Dordrecht, 2011), pp. 129-148, https://doi.org/10. 1007/978-94-007-1917-0

37. E. P. Inyang, E. P. Inyang, E. S. William, E. E. Ibekwe, and I.O.Akpan, Analytical Investigation of Meson spectrum via Exact Quantization Rule Approach, arXiv:2012.10639.

38. E.P. Inyang, E.P. Inyang, J.E. Ntibi, and E.S. William, Analytical solutions of Schrödinger equation with Kratzer-screened
Coulomb potential for a Quarkonium system, Bull. Pure Appl. Sci. 40 (2021) 14, https://doi.org/10.5958/ $2320-3218.2021 .00002 .6$

39. E.P. Inyang, E.A. Ibanga, F.A iyedun, J.E. Ntibi, and E.S. William, Non-relativistic study of generalized Yukawa potential to predict the mass-spectra of heavy quarkonium system, $J$. Nigerian Assoc. Math. Phys. 60 (2021) 3.

40. M. Abu-Shady, and H.M. Fath-Allah, The effect of extended Cornell potential on heavy and heavy-light meson masses using series method. J. Found. Appl. Phys. 6 (2019) 163.

41. E.E. Ibekwe, T.N. Alalibo, S.O. Uduakobong, A.N. Ikot and N.Y. Abdullah, Bound state solution of radial Schrödinger equation for the quark-antiquark interaction potential. Iran. J. Sci. Technol. Trans. A 44 (2020) 1191, https : / / doi.org/ 10.1007/s40995-020-00913-4

42. A. Chouikh, T. Said, and M. Bennai, Alternative Approach for Quantum Computation in a cavity QED. Quant. Phys. Lett. 6 (2017) 65, https://doi.org/10.18576/qpl/ 060109 .

43. E.E. Ibekwe, U.S. Okorie, J.B. Emah, E.P. Inyang, S.A. Ekong, Mass spectrum of heavy quarkonium for screened Kratzer potential(SKP) using series expansion method, Eur. Phys. J. Plus 136 (2021) 87, https://doi.org/10.1140/ epjp/s13360-021-01090-y

44. E. P. Inyang, E. P. Inyang, I. O. Akpan, J. E. Ntibi, and E. S. William, Analytical solutions of the Schrödinger equation with class of Yukawa potential for a quarkonium system via series expansion method, Eur. J. Appl. Phys. 2 (2020) 26, https: //doi.org/10.24018/ejphysics.2020.2.6.26.

45. E.P. Inyang, E.P. Inyang, J. Karniliyus, J.E. Ntibi, and E.S. William, Diatomic molecules and mass spectrum of Heavy Quarkonium system with Kratzer-screened Coulomb potential(KSCP) through the solutions of the Schrödinger equation, Eur. J. Appl. Phys. 3 (2021) 61, https://doi.org/10. 24018 /ejphysics.2021.3.2.61

46. Q. Dong, H.I.G. Hernandez, G-H. Sun, M. Toutounji and SH. Dong, Exact solutions of the harmonic oscillator plus nonpolynomial interaction, Proc. R. Soc. A 476 (2020) 20200050, https://doi.org/10.1098/rspa.2020.0050

47. Q. Dong, G-H. Sun, B. He and S-H. Dong, Semi-exact solutions of sextic potential plus a centrifugal term. J. Maths. Chem. 58 (2020) 2197, https://doi.org/10.1007/ s10910-020-01169-4

48. C-Y. Chan, X-H Wang, Y. You, G-H. Sun and S-H. Dong, Exact solutions of the rigid rotor in the electric field. Int. J. Quan. Chem. 120 (2020) e26336, https: / / doi.org/10.1002/ qua. 26336

49. G-H. Sun, C-Y. Chen, H. Taud, C. Yáñez-Márquez, and S-H. Dong, Exact solutions of the 1D Schrödinger equation with the Mathieu potential. Phys. Lett. A 134 (2020) 126480, https: //doi.org/10.1016/j.physleta.2020.126480

50. N. Cheemaa, A.R. Seadawy and S. Chen, Some new families of solitary wave solutions of the generalized Schamel equation and their applications in plasma physics. Eur. Phys. J. Plus 134 (2019) 117, https://doi.org/10.1140/ epjp/i2019-12467-7 
51. Q. Dong et al., Exact solutions of the sine hyperbolic type potential. J. Math. Chem. 57 (2019) 1924, https://doi. org/10.1007/s10910-019-01045-w

52. Q. Dong, G-H. Sun, M.A. Aoki, C-Y. Chen and S-H. Dong, Exact solutions of a quartic potential. Mod. Phys. Lett. A 34 (2019) 1950208, https://doi.org/10.1142/ S0217732319502080

53. Q. Dong, G-H. Sun, J. Jing and S-H. Dong, New findings for two new type sine hyperbolic potentials. Phys. Lett. A 383 (2019) 270, https://doi.org/10.1016/j. physleta.2018.10.034

54. Q. Dong et al., Semi-exact solutions of Konwent potential. Commun. Theor. Phys. 71 (2019) 231, https://doi.org/ $10.1088 / 0253-6102 / 71 / 2 / 231$

55. R. Horchani et al., Energy spectra and magnetic properties of diatomic molecules in the presence of magnetic and $\mathrm{AB}$ fields with the inversely quadratic Yukawa potential. Eur. Phys. J. D 75 (2021) 36, https: //doi.org/10.1140/epjd/ s10053-021-00038-2

56. K.J. Oyewumi, O.J. Oluwadare, K.D. Sen, and O.A. Babalola, Bound state solutions of the Deng Fan molecular potential with the Pekeris-type approximation using the Nikiforov-Uvarov (N-U) method. J. Math. Chem. 51 (2013) 976, https:// doi.org/10.1007/s10910-012-0123-6

57. C.A. Onate, M.C. Onyeaju, E. Omugbe, I.B. Okon, and O.E. Osafile, Bound state solutions and thermal properties of the modifed Tietz-Hua potential. Sci Rep 11 (2021) 2129, https : //doi.org/10.1038/s41598-021-81428-9

58. A. N. Ikot et al., Exact and Poisson Summation thermodynamic properties for diatomic molecules with shifted Tietz potential, Ind. J. Phys. 93 (2019) 1171, https://doi.org/ $10.1007 / \mathrm{s} 12648-019-01375-0$

59. U.M. Ukewuihe et al., Approximate solutions of Schrödinger equation in D Dimensions with the modified Mobius square plus Hulthen potential. Math. Comput. Sci. 2 (2021) 1, https: //doi.org/10.30511/MCS.2021.527027.1020

60. S. Dong, and S.H. Dong, Schrödinger equation with a coulomb field in 2+1 dimensions. Phys. Scr. 66 (2002) 342, https:// doi.org/10.1238/Physica.Regular.066a00342

61. C. Berkdermir, A. Berkdemir, and R.Sever, Polynomial solutions of the Schrödinger equation for the generalized WoodsSaxon potential. Phys. Rev. C 72 (2008) 027001, https: //doi.org/10.1103/PhysRevC.72.027001

62. R.L. Greene, and C. Aldrich, Variational wave functions for a screened Coulomb potential, Phys. Rev. A 14 (1976) 2363, https://doi.org/10.1103/PhysRevA.14.2363.

63. C.S. Jia, T. Chen, and L.G. Cui, Approximate analytical solutions of the Dirac equation with the generalized PöschlTeller potential including the pseudo-centrifugal term, Phys. Lett. A 373 (2009) 1621, https://doi.org/10.1016/ j.physleta.2009.03.006.

64. E.L. Hill, The Theory of Vector Spherical Harmonics, Am. J. Phys. 22 (1954) 211, https://doi.org/10.1119/1. 1933682

65. C.L. Pekeris, The Rotation-Vibration Coupling in Diatomic Molecules. Phys. Rev. 45 (1934) 98, https://doi.org/ $10.1103 /$ PhysRev.45.98
66. B.H. Yazarloo, H. Hassanabadi, and S. Zarrinkamar, Oscillator strengths based on the Mobius square potential under Schrödinger equation. Eur. Phys. J. Plus 127 (2012) 51, https://doi.org/10.1140/epjp/ i2012-12051-9

67. C.O. Edet and A.N. Ikot, Analysis of the impact of external fields on the energy spectra and thermo-magnetic properties of $\mathrm{N}_{2}, \mathrm{I}_{2}, \mathrm{CO}, \mathrm{NO}$ and $\mathrm{HCL}$ diatomic molecules. Mol. Phys. 119 (2021) e1957170, https://doi.org/10. $1080 / 00268976.2021 .1957170$

68. C. Eckart, The penetration of a potential barrier by electrons Phys. Rev. 35 (1930) 1303, https://doi.org/10.1103/ PhysRev.35.1303

69. B. J. Falaye, Any 1-state solutions of the Eckart potential via asymptotic iteration method Central Euro. J. Phys. 10 (2012) 960, https://doi.org/10.2478/ s11534-012-0047-6

70. M. Abu-Shady, Analytic solution of Dirac Equation using the Nikiforov-Uvarov method. Boson J. Mod. Phys. 1 (2015) 16.

71. C.O. Edet, and A.N. Ikot, Shannon information entropy in the presence of magnetic and Aharanov-Bohm (AB) fields. Eur. Phys. J. Plus 136 (2021) 432, https: / / doi .org/10. 1140/epjp/s13360-021-01438-4.

72. E.P. Inyang, J.E. Ntibi, E.P. Inyang, E.S. William, and C.C. Ekechukwu, Any L-state solutions of the Schrödinger equation interacting with class of Yukawa-Eckart potentials. Int. J. Innov. Sci. Eng. Technol. 7 (2020) 42.

73. S.H. Dong, W.C. Qiang, G.H. Sun, and V.B. Bezerra, Analytical approximations to the l-wave solutions of the Schrödinger equation with the Eckart potential. $J$. Phys. A 40 (2007) 10535, https: / /doi.org/10.1088/ 1751-8113/40/34/010

74. L. H. Zhang and X. P. Li, and C.S. Jia, Analytical approximation to the solution of the Dirac equation with the Eckart potential including the spin-orbit coupling term, Phys. Lett. 372 (2008) 2201, https://doi.org/10.1016/j. physleta.2007.11.022

75. H. Hellmann, A New Approximation Method in the Problem of Many Electrons, J Chem Phys 3 (1935) 61, https: //doi.org/10.1063/1.1749559

76. C. A. Onate, J. O. Ojonubah, A. Adeoti, E. J. Eweh, and M. Ugboja, Approximate Eigen Solutions of D.K.P. and KleinGordon Equations with Hellmann Potential, Afr. Rev. Phys, 9 (2014) 497.

77. S. M. Ikhdair, and R. Sever, A perturbative treatment for the bound states of the Hellmann potential, Journal of Molecular Structure: J. Mol. Struct. 809 (2007) 113, https://doi. org/10.1016/j.theochem.2007.01.019

78. M. Hamzavi, K. E. Thylwe, and A. A. Rajabi, Approximate Bound States Solution of the Hellmann Potential, Commun. Theor. Phys. 60 (2013) 1, https://doi.org/10.1088/ 0253-6102/60/1/01.

79. C. A. Onate, O. Ebomwonyi, K. O. Dopamu, J. O. Okoro, and M. O. Oluwayemi, Eigen solutions of the D-dimensional Schrödinger equation with inverse trigonometry scarf potential and Coulomb potential Chin. J. Phys, 56 (2018) 2538, https : //doi.org/10.1016/j.cjph.2018.03.013 
80. C. O. Edet et al., Solutions of Schrödinger equation and thermal properties of generalized trigonometric Poschl-Teller potential Rev. Mex. Fis. 66 (2020) 824, https://doi.org/ $10.31349 /$ RevMexFis.66.824

81. C. P. Onyenegecha et al., Approximate solutions of Schrödinger equation for the Hua plus modified Eckart potential with the centrifugal term Eur. Phys. J. Plus, 135 (2020) 571, https : //doi.org/10.1140/epjp/s13360-020-00595-2

82. B.I. Ita, Solutions of the Schrödinger equation with inversely quadratic Hellmann plus Mie-type potential using NikiforovUvarov method. Int. J. Rec. Adv. Phys. 2 (2013) 25.

83. A.R. Sari, A. Suparmi, and C. Cari, Solution of Dirac equation for Eckart potential and trigonometric Manning Rosen potential using asymptotic iteration method Chin. Phys. B, 25 (2015) 010301, https://doi.org/10.1088/ 1674-1056/25/1/010301.

84. C.A. Onate, M.C. Onyeaju, A.N. Ikot, J.O. Idiodi, and J.O. Ojonubah, Eigen solutions,Shannon entropy and fisher information under the Eckart Manning Rosen potential J. Korean Phys. Soc. 70 (2017) 339, https://doi.org/10.3938/ jkps.70.339

85. N. Hatami, J. Naji, and M. Pananeh, Analytical solutions of the Klein-Gordon equation for the deformed generalized Deng-Fan potential plus deformed Eckart potential Eur. Phys. J. Plus,134 (2019) 90, https://doi.org/10.1140/ epjp/i2019-12451-3.
86. C. Tezcan and R. Sever, A general approach for the exact solution of the Schrödinger equation Int. J. Theor. Phys. 48 (2009) 337, https://doi.org/10.1007/ s10773-008-9806-y

87. S.H. Dong, Factorization Method in Quantum Mechanics, Fundamental Theories in Physics (Springer Dordrecht, 2007), https://doi.org/10.1007/978-1-4020-5796-0

88. A.N. Ikot et al., The $\mathrm{N}$ ikiforov-Uvarov-Functional Analysis (NUFA) Method: A new approach for solving exponential-type potentials. Few-body syst. 62 (2021) 9, https : / / doi .org/ 10.1007/s00601-021-01593-5

89. O. J. Oluwadere, and K. J. Oyewumi, Energy spectra and the expectation values of diatomic molecules confined by the shifted Deng-Fan potential Eur. Phys. J. Plus 133 (2018) 422, https://doi.org/10.1140/epjp/ i2018-12210-0

90. E.P. Inyang et al., Energy spectra and expectation values of selected diatomic molecules through the solutions of Klein-Gordon equation with Eckart-Hellmann potential model. Mol. Phys. 119 (2021) e1956615, https : / / doi.org/10. $1080 / 00268976.2021 .1956615$

91. I. B. Okon, A. D. Antia, L. E. Akpabio, and B. U. Archibong, Expectation values of some diatomic molecules with Deng-Fan potential using Hellmann Feynman theorem J. Appl Phys. Sci Intl. 10 (2018) 247. 